\title{
Advances in Program Termination and Liveness
}

\author{
Byron Cook \\ Microsoft Research Cambridge
}

\begin{abstract}
Recent research advances now allow us to automatically prove termination and other liveness properties of many industrial programs. In cases where the desired property does not hold for all inputs, tools can be used to synthesize a precondition on the inputs under which the property does hold. In this tutorial I will describe these recent advances and discuss our efforts to apply termination analysis to industrial software.
\end{abstract}

